\title{
Evolutionary Algorithms Based on Decomposition and Indicator Functions: State-of-the-art Survey
}

\author{
Wali Khan Mashwani \\ Department of Mathematics, \\ Kohat University of Science \& Technology, \\ Khyber Pakhtunkhwa (KPK), Pakistan
}

\author{
Abdellah Salhi \\ Department of Mathematical Sciences, \\ University of Essex, \\ Wivenhoe Park,Colchester, UK
}

\author{
Muhammad Asif jan \\ Department of Mathematics, \\ Kohat University of Science \& Technology, \\ Khyber Pakhtunkhwa (KPK), Pakistan
}

\author{
Muhammad Sulaiman \\ Department of Mathematics, \\ Abdul Wali Khan University, Mardan, \\ Khyber Pakhtunkhwa (KPK), Pakistan
}

\author{
Rashida Adeeb Khanum \\ Department of Mathematics, \\ Jinnah College for Women Peshawar, \\ Khyber Pakhtunkhwa (KPK), Pakistan
}

\author{
Abdulmohsen Algarni \\ King Khalid University, \\ College of Computer Science, \\ Abha, Asir, Saudi Arabia
}

\begin{abstract}
In the last two decades, multiobjective optimization has become mainstream because of its wide applicability in a variety of areas such engineering, management, the military and other fields. Multi-Objective Evolutionary Algorithms (MOEAs) play a dominant role in solving problems with multiple conflicting objective functions. They aim at finding a set of representative Pareto optimal solutions in a single run. Classical MOEAs are broadly in three main groups: the Pareto dominance based MOEAs, the Indicator based MOEAs and the decomposition based MOEAs. Those based on decomposition and indicator functions have shown high search abilities as compared to the Pareto dominance based ones. That is possibly due to their firm theoretical background. This paper presents state-of-the-art MOEAs that employ decomposition and indicator functions as fitness evaluation techniques along with other efficient techniques including those which use preference based information, local search optimizers, multiple ensemble search operators together with self-adaptive strategies, metaheuristics, mating restriction approaches, statistical sampling techniques, integration of Fuzzy dominance concepts and many other advanced techniques for dealing with diverse optimization and search problems
\end{abstract}

Keywords-Multi-objective optimization, Multi-objective Evolutionary algorithms (MOEAs), Pareto Optimality, Multi-objective Memetic Algorithm (MOMAs), Pareto dominance based MOEA, Decomposition based MOEA, Indicator based MOEAs.

\section{INTRODUCTION}

Multi-objective optimization deals with problems involving two or more conflicting objectives. In general, optimization problems can be combinatorial or continuous. The Traveling Salesman Problem (TSP) [165] and the Minimum Spanning Tree (MST) are two well-known combinatorial problems. Combinatorial optimization has various applications [38], [35], [170], [31], [181] in air traffic routing, the design of telephone networks, electrical engineering, hydraulic networks, cable TV and computer systems and others. Continuous optimization is widely used in mechanical design problems [109]. This study is concerned with multi-objective optimization problems (MOPs) including continuous variables. The general formula- tion of a MOP is:

$$
\begin{aligned}
& \text { minimize } F(x)=\left(f_{1}(x), \ldots, f_{m}(x)\right)^{T} \\
& \text { subject to } x \in \Omega
\end{aligned}
$$

where $\Omega$ is the decision space, $x=\left(x_{1}, x_{2}, \ldots, x_{n}\right)^{T}$ is a decision vector and $x_{i}, i=1, \ldots, n$ are decision variables, $F(x): \Omega \rightarrow R^{m}$ includes $m$ real valued objective functions in the objective space $R^{m}$. If $\Omega$ is a closed and connected region in $R^{n}$ and all objective functions involve continuous variables then problem (1) is called a continuous MOP.

In real world multi-objective optimization problems, objective functions are usually in conflict or mostly incommensurable. Consequently, there is not a unique solution that can minimize all the objective functions at the same time. The problem must be solved in terms of Pareto optimality. This concept was first devised by Francis Ysidro Edgeworth in 1881 and then later on generalized by Vilfredo Pareto in 1896 . To describe this concept, we will introduce a few definitions.

A solution $u=\left(u_{1}, u_{2}, \ldots, u_{n}\right) \in \Omega$ is said to be Pareto optimal if there does not exist another solution $v=$ $\left(v_{1}, v_{2}, \ldots, v_{n}\right) \in \Omega$ such that $f_{j}(u) \leq f_{j}(v)$ for all $j=$ $1, \ldots, m$ and $f_{j}(u)<f_{j}(v)$ for at least index $k$. An objective vector is Pareto optimal if the corresponding decision vector is Pareto optimal. All the Pareto optimal solutions in the decision space form the Pareto Set (PS) and their image in the objective space forms a Pareto Front (PF), [136], [37], [41].

In the last few years, several multi-objective evolutionary algorithms (MOEAs) have been developed [98], [84], [123], [127], [189], [167], [85], [121] and they have proven their power in many demanding real-world optimization tasks [36], [35], [31], [123], [189], [127], [96]. Classical MOEAs can generally be divided into three main paradigms: the Pareto dominance based MOEAs [42], [193], [192], [149], [65], the indicator based evolutionary algorithms (IBEAs), [199], [20], [12], [19], [157], [178] and the decomposition based MOEAs [183], [101], [185], [129], [132], [125], [86], [130]. MOEAs operate on a population and approximate the set of optimal solutions in a single simulation run, maintaining diversity 
among these solutions using different measures such as fitness sharing techniques, the niching approach, the Kernel approach, the nearest neighbour approach, the histogram technique, the crowding/clustering estimation technique, the relaxed form of dominance and the restricted mating and many others.

The fast Non-dominated Sorting Genetic Algorithm II (NSGA-II), [42], SPEA2 [192], the Pareto Archive Evolution Strategy (PAES), [88], the Multi-Objective Genetic Algorithm (MOGA), [52], and the Niched Pareto Genetic Algorithm (NPGA), [65], are well known Pareto dominance based MOEAs. Among them, NSGA-II [42] is an improved version of the Non-dominated Sorting Genetic Algorithm (NSGA), [80] for dealing with MOPs. It generates offspring with crossover and mutation and selects the next generation according to non-dominated sorting and crowding distance comparison. SPEA2 [192] is an improved version of Strength Pareto Evolutionary Algorithm (SPEA), [194]. SPEA2, [192], incorporates a fine-grained fitness assignment strategy, a density estimation technique, and an enhanced archive truncation method in contrast to SPEA [194]. It incorporates a mechanism like k-Nearest Neighbour ( $\mathrm{kNN}$ ) and a specialized ranking system to sort the members of the population, and select the next generation of population, from combination of current population and offsprings population created by crossover and mutation. Both SPEA2 [192] and NSGA-II [42] have shown excellent performances on various real-world, scientific and engineering problems.

Memetic Algorithms (MAs) are a growing area of research motivated by the meme concept introduced by Richard Dawkins. MAs are hybrid algorithms that combine local search optimizers and genetic algorithms for solving NPhard problems. The first multi-objective MA was developed by Ishibuchi and Murata [67]. That was then improved by Jaszkiewicz, [1], [77]. Basically, these algorithms reformulate the given MOP as simultaneous optimization of all weighted Tchebycheff functions or all weighted sum functions. The Adaptive Multi-objective optimization using Genetically Adaptive Multimethod search (AMALGAM) [177] blends multiple search operators to evolve populations.

This paper provides a state-of-the-art survey of MOEAs that employ indicator and decomposition functions for guiding their search and evolve their populations. We have included, especially, those approaches which are recently developed and found in the existing specialized literature of the evolutionary computation (EC).

The rest of this paper is organized as follows. Section II provides the latest and enhanced variants of MOEA/D. Section III is related to Indicator based EAs. Section IV will finally conclude this paper with some future research directions.

\section{Decomposition Based Multi-objective EVOLUTIONARY AlgORITHM}

Decomposition is a procedure that break down the given system or task into smaller pieces and then optimize them either sequentially or parallel [112]. This concept is already incorporated in many meta-heuristics, namely, tabu search technique [54], simulated annealing (SA) [175], ant colony optimization (ACO) [58], differential evolution [103], particle swarm optimization (PSO) [156], genetic algorithm (GA)
[43], evolutionary strategy (ES) [102] for solving various test suits of optimization and search problems. Metaheuristics are higher-level procedure or heuristics designed that efficiently provide good set of solutions for the given optimization problems [22], [169].

In [180], a decomposition-based multi-objective differential evolution particle swarm optimization (DMDEPSO) is developed and intelligently resolved the problems of design of a tubular permanent magnet linear synchronous motor (TPMLSM).Two-Phase Local Search (TPLS) is proposed in [155] in order tackle TSP with bi-objective functions. This proposed algorithm in their first phase of optimization process generates good initial solution based on single objective function and then forward that solutions to their second phase, where local search technique is used to apply on them by using aggregation of objective functions till the time no optimum solutions are found. Improved TPLS are devised in [46], [47] aiming at to improve its anytime performance by employing regular distribution of the weight vectors in order to equally distribute the effort of the objective space in all directions. In [142], [141], cellular multi-objective genetic algorithm (CMOGA) is suggested. It uses canonical cellular model of GAs (cGA) as a baseline model and habituating the weighted sum approach in order to convert the MOP at hand into scalar optimization problems. Moreover, CMOGA also implanted in multi-objective genetic algorithm (MOGA) framework the cellular structures for residing each individual of its population in a cell of spatially structured space. It then locally utilizes genetic operations in the neighbourhood of each cell for creating an offspring population. Another novel and promising cellular genetic algorithm called MOCell developed in [144] for dealing with multi-objective continuous optimization problems. This algorithm maintain an external archive for storing non-dominated set of solutions and utilize them again at certain stages of population evolution. Another algorithm of same nature was proposed in [3] which does not adopt an external archive as like MOCell does [144] in their algorithmic framework.

Nowadays, memetic algorithms (MAs) is an another emerging and hot area of research inspired by Darwinian principles of natural evolution and Dawkins' notion of a meme [61], [94]. This was first introduced by Pablo Moscato in 1989 [138]. They are also called Baldwinian evolutionary algorithms (EA), Lamarckian EAs [150], cultural algorithms, genetic local search or hybrid algorithm [139], [147], [146], [151]. This class of algorithms have shown great performance and achievements in solving real-world problems and various complicated test suites of MOPs [140], [60], [68], [53], [137], [126], [128].

Ishibuchi and Murata were the first researchers whose proposed the multi-objective genetic local search (MOGLS) [70], [67] for solving multi-objective combinatorial optimization problems. This proposed methodology has utilized the scalar fitness function with random weights generation strategy to guide their population until stoping criteria not satisfied. Jaszkiewicz has further improved MOGLS [78] by incorporating the modified parent selection mechanism in Ishibuchi's MOGLS. Jaszkiewicz examined the performance of their MOGLs using test suit of multiple-objective 0/1 knapsack problems (MOKPs). Later on, Ishibuchi further enhanced its MOGLS [63], here the authors apply local search upon limited 
number solutions as per probability value, $P_{L S}$ with aim to minimize the computation burden of the existing Ishibuchi's MOGLS [70], [67].

Multi-objective evolutionary algorithm based on decomposition (MOEA/D) [183] is well-known developed paradigm in evolutionary computation field. This novel and robust stochastic technique bridges traditional mathematical programming and evolutionary computing (EC) and transforms the MOP at hand into $N$ different scalar optimization sub-problems (SOPs). For this purposes, it employs an aggregation based techniques including weighted sum function, Tchebycheff approach and many others [136] and then optimizes all these SOPs simultaneously rather than solving a MOP directly. Aggregation based fitness assignment strategy of MOEA/D naturally handles convergence and diversity issues contrary to non-decomposition based approaches. The main feature of MOEA/D is its neighborhood relationships among their subproblems that defines based on the distances between their aggregation coefficient vectors.

The original MOEA/D was then further enhanced in [101] by replacing simulated binary crossover (SBX) [81] with differential evolution (DE) [162]. Moreover, in this improved version of MOEA/D called MOEA/D-DE [101], two neighbourhoods are used and each child solution to replace minimum number of old solutions in its neighbourhood structure. Moreover, general guidelines for the formulation of multiobjective continuous test instances are also main part of this study [101]. Recently, in [32], multiple neighbourhood replacement strategies, reference point determination and different decomposition methods are explicitly analyzed upon multi-objective flowshop scheduling test problems. Dynamical resources allocation scheme for the subproblems are introduces in [185], where each subproblems get resources in dynamical manner based on suggested utility function.Gaussian process model is integrated in MOEA/D framework and as result MOEA/D-EGO is developed in [186] to handle an expensive MOP by converting into a number of single-objective optimization subproblems. Gaussian process models provides a probabilistic non-parametric modelling approach for black-box identification of non-linear dynamic systems. The Gaussian processes can highlight areas of the input space, where prediction quality is poor, due to the lack of data or its complexity, by indicating the higher variance around the predicted mean [90].

In [72], [71], [73], [74], simultaneous use of aggregation functions along with neighborhood structures are incorporated in an original MOEA/D framework [183], [101] for solving combinatorial optimization problems with many objective functions. In [154], each subproblem have been associated more than one solution to maintain search diversity.

Mostly MOEA/D [183] frameworks use Tchebycheff and the weighted sum approaches with fixed weight vectors strategy. However, in [99], the wights of aggregation functions are adjusted in adaptive as well as fixed manners. This algorithm make use of external archive and stores non-dominated solutions using modified $\epsilon$-dominance strategy and to utilize that solutions in the generating process of weight vectors. The use of fixed weight vectors adjustment is sometimes creates hurdles that are expected in solving problems with complicated Pareto fronts (PFs), discontinuity or sharpness or low tail in their structure. This novel procedure for adaptive weight vectors generation adopted in MOEA/D-AWA: MOEA/D-adaptive weight adjustment have offered promising results coping with MOPs used in [161].

Aggregation approaches like weighted sum approach [136] and the Tchebycheff approach [136] are widely employed in the framework of MOEA/D. However, they are sometimes unable to deal with problems having had disparately scaled objective functions. In [163], Normal Boundary Intersection (NBI) style Tchebycheff approach is utilized in MOEA/DDE [101] as resultant new algorithm called MOEA/D-NBI has been developed for solving portfolio management MOPs. The same NBI-style Tchebycheff approach is also implemented in [184] for also dealing with portfolio management problems.

In [97], simulated annealing (SA) is integrated in MOEA/D and the combined impact analyzed upon both constrained multi-objective knapsack problems and unconstrained multiobjective traveling salesman problem. In this algorithm, simulated annealing (SA) has been used as local search with adaptive procedures.

A novel smart multi-objective particle swarm optimization based decomposition (SDMOPSO) is recently suggested in [2] for solving ZDT test problems [198]. Noting that different only decomposition strategies hare incorporated particle swarm optimization (PSO) [49] framework. Like MOEA/D, this algorithm transforms the given MOP into $N$ numbers of SOPs. In [111], PSO injected to MOEA/D to handle the multiobjective 0/1 knapsack problems and also continuous multiobjective optimization problems. In [125], [132], differential evolution [162] and particle swarm optimization [49] are incorporated simultaneously with self-adaptive procedures in MOEA/D [183] to handle five standard ZDT test problems [198] and CEC'09 test instances [187].

In [33], two enhanced mechanisms such as guided mutation and priority update schemes are introduced in the original framework MOEA/D [101]. This algorithm was denoted by MOEA/D-GM and efficiently tackled CEC'09 test instances [187] consist of both unconstrained and constrained MOPs. MOEA/D-GM creates its new population with guided mutation (GM) rather than differential evolution (DE) [162] and also introduces update mechanism based on priority queue of subproblems.

An interactive decomposition based multiobjective evolutionary algorithm (IMOEA/D) is recently proposed in [55] that incorporates preference mechanism for selecting the preferred sub-problems rather than the preferred region in the objective space. At each interaction, iMOEA/D provides a set of current solutions to decision maker (DM) to pick out the most preferred one for guiding search towards the neighborhood of the selected ones. iMOEA/D are tested upon benchmark functions and various utility functions are used to simulate the DM's responses. Iterative threshold based MOEA/D framework developed in [100] for optimizing sparse signal recovery in compressive sensing.

The synthesis problem of the difference patterns of monopulse antenna arrays are modeled as MOP in [153] with composed of two objective functions including the maximum side-lobe level (MSLL) and beam width (BW) of principal 
lobe. MOEA/D-DE [101] is applied on these modeled problems and approximated their Pareto Fronts (PFs) with different number of elements and sub-arrays. Linear antenna array design is an electromagnetic optimization problems and can be formulated as a MOP with two objectives: the minimum average side lobe level (SLL) and null control in specific directions. MOEA/D-DE [101] is also applied on these problems as well and for it an optimized spacing between the elements of linear array while achieving the best possible trade-off between the above mentioned two design objective functions [152]. Furthermore, MOEA/D-DE [101] is applied on problems formulated in [57] and have found better optimally sized two mixed-mode circuits including positive second generation current conveyor and current feedback operational amplifier as compared to NSGA-II [42].

A thread-based parallel implementation of MOEA/D framework is devised recently in [145], [48] by executing on modern multi-core processors. Parallel Decomposition (PaDe) is recently developed in [117]. It has habituated a asynchronous generalized island model for solving various decomposed problems. In [164], a parallel version of MOEA/D has been developed that assigns the computational resources for generating solutions in the minimum overlapped update ranges of solutions and tournament selection based on the scalarizing function value to strengthen the selection pressure of its parent population. A new fine grained message passing schemes for the distribution the MOEA/D computations are implemented in MOEA/D-MP framework [44]. In [119], new selection and replacement strategies have been adopted in MOEA/D for solving bi-objective combinatorial optimization problems.

In [86], [129], [84], [85], different multiple search operators are being engaged in MOEA/D framework [185] and handled the test instances designed for the special session of MOEAs competition in IEEE Congress of Evolutionary Computation (CEC'09) [187]. Two different structured and well-established MOEAs in evolutionary computing (EC) field, namely, MOEA/D [183] and NSGA-II [42] are combined at population and generation levels in [124], [122] for two different benchmark functions expressed in continuous variables. The same two well-known algorithms are also engaged altogether for dealing with a hard multiobjective optimization problem in [134]. The concepts of fuzzy dominance are recently introduced in the MOEA/D framework [143] for enhancement of MOEA/D paradigm. The impact of the ensemble use of the different neighbourhood sizes are recently investigated in [188] based on self-adaptive procedures. Very recently, the impact of multiple crossovers are examined in [131] using MOEA/D-DRA [185] as global search technique and experiment carried over CEC'09 test instances [187].

In [4], Tabu Search (TS) is used within MOEA/D framework to solve the multiobjective permutation flow shop scheduling problems. In [91], several problem-specific operators are also investigated in MOEA/D framework [101] to tackle multiobjective mobile agent routing problems. An encoding representation and various genetic operators are designed for wireless sensor networks deployment and power assignment problems (DPAPs) in [92]. Several constraint handling techniques are also adopted in the framework of MOEA/D algorithmic structure for solving constrained Kconnected DPAP in WSNs [93]. Covariance matrix adaption evolution strategy (CMA-ES) has been injected to MOEA/D as a local search optimizers. The resultant algorithm abbreviated as MOEA/D-CMA developed in [182] for handling the CEC'09 box-constrained benchmark functions [187].

In [82], Ant colony optimization (ACO) is incorporated within MOEA/D framework [183] and an algorithm called MOEA/D-ACO is developed, where the effects of grouping, neighborhood, and the location information of current solutions are explicitly analyzed over multiobjective $0 / 1$ knapsack test problems. In [190], a generator based on multivariate Gaussian models is engaged in MOEA/D framework, where probability models samplings new trial solutions and Gaussian distribution models extracts both local and global population distribution information in robust manners. An efficient multiobjective memtic algorithm called MOMADA is recently developed in [83] by utilizing modified Pareto local search methods [113] to explore the neighborhoods of different locally optimal solutions of the subproblem. In [120], Nelder and Meads algorithm also known as nonlinear simplex search method has employed as local search optimizer in MOEA/D framework for solving Zitzler-Deb-Thiele (ZDT) [198] and Deb-Thiele Laumanns-Zitzler (DTLZ) benchmark functions [40].

Decomposition-based memetic algorithm with extended neighbourhood search (D-MAENS) is developed in [135] for solving multi-objective capacitated arc routing problem (MOCARP). D-MAENS also decomposes the given MO-CARP as like MOEA/D [183] into a number of scalar subproblems by employing the weighted sum approach with a set of uniformly distributed weight vectors adjustment. A new replacement mechanism and the assignment mechanism for offspring solutions are introduced in improved D-MAENS [166]. In [160], a decomposition based memetic algorithm is proposed and examined their performance upon multiobjective vehicle routing problem with time windows (MOVRPTW). The suggested algorithm accommodates three types of local search methods periodically in combination with novel selection operator. In [132], [125], multi-objective memetic algorithm based on decomposition is developed for solving multi-objective continuous optimization problems. The proposed algorithm employs particle swarm optimization (PSO) and deferential evolution (DE) with self-adaptive manner for population evolution in their suggested enhanced MOEA/D version.

In [133], artificial bee colony (ABC) and teaching-learningbased optimization (TLBO) are engaged within MOEA/D framework to tackle the ZDT test problems [198] and seven unconstrained MOPs of the test suite of the 2009 IEEE congress on evolutionary computation [187]. ABC [62] works on the foraging behavior of a honey bee and TLBO [148] works on the philosophy of teaching and learning process. Oppositionbased learning is a fast growing area of research developed in [172]. OBL has been incorporated in MOEA/D framework as resultant an algorithm called MOEA/D-OBL has been sprang up recently in [115]. The suggested opposition-based initial population and opposition-based learning strategy to generate an offspring population have improved the convergence ability of original MOEA/D [183]. An improved MOEA/D algorithm denoted by TMOEA/D is developed in [110]. The proposed algorithm has utilized a monotonic increasing function and transformed each individual objective function into the one as 
resultant the curve shape of the non-dominant solutions of the transformed multi-objective problem get closed to the hyperplane whose intercept of coordinate axes is equal to one in the original objective function space.

In [6], [5], a decomposition based evolutionary algorithm is developed for solving both benchmark functions with many objectives and also real-world problems including the car side impact problem, the water resource management problem and the constrained ten-objective general aviation aircraft (GAA) design problem. Moreover, the proposed algorithm have been employed Latten hypercube sampling (LHS) mechanism for reference points generation and adaptive epsilon scheme to establish balance between convergence vs diversity dilemma.

MOEA/D with uniform decomposition measurement (MOEA/D-UDM) developed in [116] for many-objective problems (MAPs). In MOEA/D-UNM [116], the authors have been highlighted two main issues concerned with original MOEA/D paradigm dealing with MAPs, firstly, the number of constructed weight vectors are not arbitrary and mainly distributed on the boundary of weight space; secondly, the relationship between the optimal solution of subproblem and its weight vector is nonlinear for the Tchebycheff decomposition approach. To address aforementioned issues, a novel weight vectors initialization method based on the uniform decomposition measurement and modified Tchebycheff decomposition function have introduced in the MOEA/D-UNM framework while coping with MAPs. In [105], both dominance and decomposition concepts have been combined in order to exploit the merits of both paradigms for the purposes to maintain balance between convergence and diversity in the process of population evolution while coping with MAPs.

The concepts of bandit-based operator selection (AOS) method and fitness-rate-rank-based multiarmed bandit (FRRMAB) are borrowed from the existing literature and have been incorporated in MOEA/D framework as consequence MOEA/D-FRRMAB is developed in [106] to handle manyobjective optimization problems. A stable matching model based on the preference articulations is employed in [108] as a resultant an algorithm called MOEA/D-STM is developed. The only difference in MOEA/D-STM and [107] are, MOEA/DSTM considers the perpendicular distance between $x$ and the weight vector of subproblem $p$. However, the algorithm suggested in [107] make use of niche count of $p$ is an additional term with perpendicular distance measurement for diversity improvement and promotion in their current population. MOEA/D-STM uses a stable matching model to find a suitable matching between subproblems and solutions.In [107], the selection of an appropriate solution for each subproblem is based on the interrelationship between subproblems and solutions.

Hybrid MOEA is developed in [104] which maintain different selection principles and two separate co-evolving archives to hold non-dominated solutions. One archive stores solutions with competitive selection pressure and other preserves a population with a satisfied distribution in the objective space. Furthermore, to exploit guidance information towards the Pareto-optimal set (PS), a restricted mating selection mechanism is employed in this algorithm for selecting mating parents from each archive to produce an efficient offspring solutions. Recently, [130], multiobjective algorithm based on multi-method is developed. MMTD employs two well-known algorithms, MOEA/D [183] and NSGA-II [42], for population evolution for dealing with CEC'09 test instances [187] and ZDT test problems.

\section{INDICATOR BASED EVOLUTIONARY ALGORITHM}

Hypervolume metric was first introduced by Zitzler and Thiele as an indicator or measurement function. It measures both convergence and diversity which are desirable in the context of multi-objective evolutionary optimization [197], [193], [195]. It measures volume dominated by non-dominated solutions in objective space and extremely suitable for assessing the dominance levels of multiple set of solutions in multiobjective evolutionary optimization. For example, consider two Pareto sets (i.e. Aand $B$ ), then the hypervolume indicator values of set $A$ will be higher than $B$ if $A$ dominates $B$. Hypervolume is also called $S$ metric or size of the space covered [191] or Lebesgue measure [95], [50]. It has been used as part of the selection or archiving process of MOEAs.

In the recent few years, the indicator based evolutionary algorithms (IBEAs) have gained growing attention and much popularity due to its strong theoretical support and background [30], [11], [26], [7], [10]. They have shown high search ability in various investigations [179], [13], [17]. The main features of these algorithms are, they do not need any diversity maintenance mechanism because indicator functions are automatically recover the issue of diversity promotion among their population solutions [176]. The first Hypervolume indicator based EAs called "hypervolume by slicing objectives (HSO)" was developed by Eckart Zitzler in his seminal work [191] and also seminal work of J.Knowels [45]. Subsequently, various indicator based evolutionary algorithms were developed by employing various procedures as like preferences based information [34], [25], [171], [8], [9], [196], different local search optimizers and many others [158]. One practical drawbacks of IBEA is that it needs much time for hypervolume calculation while dealing with many objectives problems. To address the mentioned drawbacks, in the recent past several faster and enhanced of IBEAs have been developed for solving both continuous and combinatorial optimization problems [114], [24], [173], [14], [28]. The algorithms developed in [114], [24] use objectives in place of points and as resultant they are quickly determine their solution contribution to the front. A methodology based on Monte Carlo sampling simulation has been recently introduced in [14] to estimate the ranks of their individuals induced by the hypervolume indicator for not determining the exact indicator values. In [15], [16], HypE: hypervolume estimation algorithm is devised for multiobjective Optimization. HypE uses the same Monte Carlo simulation method to approximate the exact hypervolume values. A fast and enhanced version of HypE is developed [18] for solving many objectives problems. In [29], a generalized methodology for preference-directed hypervolume-based multi objective search called W-HypE is developed. W-HypE also relies on Monte Carlo sampling and thereby allows to tackle problems with an arbitrary number of objectives.

In [76], an idea of scalarizing function-based hypervolume approximation method are introduced IBEAs framework to dealt with many objectives optimization problems. A simple and fast hypervolume indicator-based MOEA (FV-MOEA) is 
proposed [79] that selects partial solutions rather than the whole solution set of solutions and quickly update the exact hypervolume contributions of different solutions. An iterative approach to indicator-based evolutionary multiobjective optimization is proposed in [69] with main feature in which only a single solution is obtained within single run. The proposed algorithm needs multiple runs for finding a set of solutions.

In [21], an indicator based evolutionary algorithm (IBEA) are compared with well-known algorithms including a fast nondominated sorting genetic algorithm II (NSGA- II) [42] and strength Pareto evolutionary algorithm (SPEA-II) [192] over standard portfolio optimization problems. An algorithm called Prospect indicator based evolutionary algorithm (PIBEA) is developed in [23] for solving DTLZ MOPs [40].The algorithmic structure of PIBEA is somewhat similar to well-known NSGA-II framework, however, it measures the potential of each individual to reproduce offspring that dominate itself and spread out in the objective space of the given MOPs. In [168], a directed search (DS) is incorporated as local search method within global indicator based optimization algorithms. In [89], Newton steepest descent method [51] and Hooke \&Jeeves [64] have been concurrently integrated within SMS-EMOEA [20] framework to handle ZDT test problems [198].

An indicator-based ant colony optimization algorithm which is abbreviated as IBACO is devised [118] for solving the multi-objective knapsack problem (MOKP). IBACO engages binary quality indicators to reinforce their best solutions and not to eliminate the worst ones as exercised in the selection phase of IBEA [200].

An indicator-based EMOA called R2-IBEA is proposed in [157], [174], [27] for solving both ZDT [198] and DTLZ test problems [39]. R2-IBEA eliminates dominance ranking and performs selection with the $R 2$ indicator [59].The $R 2$ indicator usually requires a set of weight vectors that are uniformly distributed in the objective space. R2-IBEA is similar to MOEA/D [183] in a sense, it also habituates aggregation function like the Tchebycheff function with uniformly distributed weight vectors. R2IBEA [157] dynamically adjusts the location of the reference point according to the extent of the current generation individuals in the objective space. The key feature of $R 2$ indicator is that it is computationally much less expensive than the hypervolume indicator. Another IBEA that avoids dominance ranking and uses a binary $\epsilon_{2}$ indicator in the selection process of suggested $I B E A-\epsilon_{2}$ [199]. The $\epsilon_{2}$ is also called binary hypervolume indicator which is also computational cost exponentially grows deal with problems having many objective functions.

In [66], the algorithmic behaviors of MOEAs belongs to Pareto dominance-based, decomposition based and hypervolume-based categories are experimentally analyzed upon many-objective knapsack problems. The gathered experimental results are clearly indicate the superiority of decomposition based MOEAs against Pareto dominance and indicators based EAs dealing with knapsack benchmark functions with many objectives.

\section{CONCLUSION}

Pareto dominance-based EAs are main streams in the field of evolutionary computation (EC). However, their perfor- mance are greatly degraded on many objectives problems [87], [159], [56], [75]. Indicator based and decomposition based EAs are promising paradigms of EC. Indicator based EAs (IBEAs) mostly use the hypervolume as the indicator function to guide their process of population evolution. Decomposition based MOEAs use aggregation approaches as fitness functions and neighbourhood relationship to structure their scalar optimization problems (SOPs). MOEA/D uses several aggregation functions (i.e., weighted sum approach, Tchebycheff approach and normal-boundary intersection method and the normalized normal constraint method) for converting the problem of approximating the PF into a number of scalar optimization problems functions. This paper provides the latest review of MOEAs that integrate the decomposition concept and indicator functions in their framework along with other efficient techniques like the incorporation of preference based information, local search optimizers, multiple search operators with self-adaptive strategies, metaheuristics, mating restriction approaches, statistical sampling techniques, Fuzzy dominance concepts to tackle issues of convergence and diversity in an efficient manner for dealing with different real-world problems and diverse and complicated MOPs test suites.

\section{REFERENCES}

[1] A.Jaszkiewicz, "Genetic local search for multi-objective combinatorial optimization," European Journalof Operational Research, vol. 137, no. 1, pp. 50-71, 2002.

[2] N. Al Moubayed, A. Petrovski, and J. McCall, "A Novel Smart Multi-objective Particle Swarm Optimisation Using decomposition,” in Proceedings of the 11th international conference on Parallel problem solving from nature: Part II, ser. PPSN'10. Berlin, Heidelberg: Springer-Verlag, 2010, pp. 1-10.

[3] E. Alba, B. Dorronsoro, F. Luna, A. J. Nebro, P. Bouvry, and L. Hogie, "A Cellular Multi-Objective Genetic Algorithm For Optimal Broadcasting Strategy In Metropolitan MANETs," Computer Communications, vol. 30, no. 4, pp. 685-697, 2007.

[4] A. Alhindi and Q. Zhang, "MOEA/D with Tabu Search for Multiobjective Permutation Flow Shop Scheduling Problems," in IEEE Congress on Evolutionary Computation (CEC'14), 2014, pp. 1155-1164.

[5] M. Asafuddoula, T. Ray, and R. Sarker, "A Decomposition-Based Evolutionary Algorithm for Many Objective Optimization," IEEE Transactions on Evolutionary Computation, vol. 19, no. 3, pp. 445460, 2015.

[6] _ "A Decomposition Based Evolutionary Algorithm for Many Objective Optimization with Systematic Sampling and Adaptive Epsilon Control," in Evolutionary Multi-Criterion Optimization, ser. Lecture Notes in Computer Science, R. Purshouse, P. Fleming, C. Fonseca, S. Greco, and J. Shaw, Eds. Springer Berlin Heidelberg, 2013, vol. 7811, pp. 413-427.

[7] A. Auger, J. Bader, and D. Brockhoff, "Theoretically Investigating Optimal $\mu$-Distributions for the Hypervolume Indicator: First Results For Three Objectives," in Conference on Parallel Problem Solving from Nature (PPSN XI), ser. LNCS, R. Schaefer et al., Eds., vol. 6238. Springer, 2010, pp. 586-596.

[8] A. Auger, J. Bader, D. Brockhoff, and E. Zitzler, “Articulating User Preferences in Many-Objective Problems by Sampling the Weighted Hypervolume," in Genetic and Evolutionary Computation Conference (GECCO 2009), G. Raidl et al., Eds. New York, NY, USA: ACM, 2009, pp. 555-562.

[9] _ - "Investigating and Exploiting the Bias of the Weighted Hypervolume to Articulate User Preferences," in Genetic and Evolutionary Computation Conference (GECCO 2009), G. Raidl et al., Eds. New York, NY, USA: ACM, 2009, pp. 563-570.

[10] — - "Hypervolume-based Multiobjective Optimization: Theoretical Foundations and Practical Implications," Theoretical Computer Science, vol. 425, pp. 75-103, 2012. 
[11] — "Hypervolume-Based Multiobjective Optimization: Theoretical Foundations and Practical Implications," Theoretical Computer Science, vol. 425, pp. 75-103, 2012.

[12] J. Bader, "Hypervolume-Based Search for Multiobjective Optimization: Theory and Methods," Ph.D. dissertation, ETH Zurich, Switzerland, 2010.

[13] _ _ "Hypervolume-Based Search for Multiobjective Optimization: Theory and Methods," Ph.D. dissertation, ETH Zurich, Switzerland, 2010.

[14] J. Bader, K. Deb, and E. Zitzler, "Faster Hypervolume-based Search using Monte Carlo Sampling," in Conference on Multiple Criteria Decision Making (MCDM'08), ser. LNEMS, M. Ehrgott et al., Eds. vol. 634, Heidelberg, Germany, 2010, pp. 313-326.

[15] J. Bader and E. Zitzler, "HypE: An Algorithm for Fast HypervolumeBased Many-Objective Optimization," Computer Engineering and Networks Laboratory (TIK), ETH Zurich, TIK Report 286, November 2008.

[16] _ "HypE: An Algorithm for Fast Hypervolume-Based ManyObjective Optimization," Computer Engineering and Networks Laboratory (TIK), ETH Zurich, TIK Report 286, 2008.

[17] _ , "Robustness in Hypervolume-based Multiobjective Search," Computer Engineering and Networks Laboratory (TIK), ETH Zurich, TIK Report 317, 2010

[18] _- "Hype: An algorithm for fast hypervolume-based manyobjective optimization," Evolutionary Computing, vol. 19, no. 1, pp. 45-76, Mar. 2011.

[19] — "HypE: An Algorithm for Fast Hypervolume-Based ManyObjective Optimization," Evolutionary Computation, vol. 19, no. 1, pp. 45-76, 2011.

[20] N. Beume, B. Naujoks, and M. Emmerich, "SMS-EMOA: Multiobjective Selection based on Dominated hypervolume," European Journal of Operational Research, vol. 181, no. 3, pp. 1653-1669, 2007.

[21] S. Bhagavatula, S. Sanjeevi, D. Kumar, and C. Yadav, "Multi-objective indicator based evolutionary algorithm for portfolio optimization," in Advance Computing Conference (IACC), 2014 IEEE International, 2014, pp. 1206-1210.

[22] L. Bianchi, M. Dorigo, L. Gambardella, and W. Gutjahr, "A Survey on Metaheuristics for Stochastic Combinatorial Optimization," Natural Computing, vol. 8, no. 2, pp. 239-287, 2009.

[23] P. Boonma and J. Suzuki, "PIBEA: Prospect Indicator Based Evolutionary Algorithm for Multiobjective Optimization Problems.'

[24] L. Bradstreet, L. While, and L. Barone, "A fast incremental hypervolume algorithm," IEEE Transactions on Evolutionary Computation, vol. 12 , no. 6 , pp. $714-723,2008$.

[25] J. Branke, K. Deb, K. Miettinen, and R. Slowinski, Eds., Multiobjective Optimization: Interactive and Evolutionary Approaches. Springer, 2008.

[26] D. Brockhoff, T. Friedrich, and F. Neumann, "Analyzing Hypervolume Indicator Based Algorithms," in Conference on Parallel Problem Solving From Nature (PPSN X), ser. LNCS, G. Rudolph et al., Eds., vol. 5199. Springer, 2008, pp. 651-660.

[27] D. Brockhoff, T. Wagner, and H. Trautmann, "R2 Indicator Based Multiobjective Search," Evolutionary Computation Journal, vol. 23, pp. 369-395, 2015

[28] D. Brockhoff and E. Zitzler, "Improving Hypervolume-based Multiobjective Evolutionary Algorithms by Using Objective Reduction Methods," in Congress on Evolutionary Computation (CEC 2007). IEEE Press, 2007, pp. 2086-2093.

[29] D. Brockhoff, J. Bader, L. Thiele, and E. Zitzler, "Directed Multiobjective Optimization Based on the Weighted Hypervolume Indicator,' Journal of Multi-Criteria Decision Analysis, vol. 20, no. 5-6, pp. 291317, 2013.

[30] D. Brockhoff, T. Friedrich, and F. Neumann, "Analyzing Hypervolume Indicator Based Algorithms," in Proceedings of the $10^{\text {th }}$ International Conference on Parallel Problem Solving from Nature: PPSN X. Berlin, Heidelberg: Springer-Verlag, 2008, pp. 651-660.

[31] Carlos and R. L. Becerra, "Evolutionary Multi-Objective Optimization in Materials Science and Engineering," Materials and Manufacturing Processes, vol. 24, no. 2, pp. 119-129, 2009.
[32] P. C. Chang, S. H. Chen, Q. Zhang, and J. L. Lin, "MOEA/D for Flowshop Scheduling Problems," in IEEE Congress on Evolutionary Computation, 2008, pp. 1433-1438.

[33] C.-M. Chen, Y.-P. Chen, and Q. Zhang, "Enhancing MOEA/D with Guided Mutation and Priority Update for Multi-Objective Optimization," in Proceedings of the IEEE Congress on Evolutionary Computation, CEC 2009, Trondheim, Norway, 18-21 May, 2009, 2009, pp. 209-216.

[34] T. Chugh, K. Sindhya, J. Hakanen, and K. Miettinen, "An Interactive Simple Indicator-Based Evolutionary Algorithm (I-SIBEA) for Multiobjective Optimization Problems," in Evolutionary Multi-Criterion Optimization, ser. Lecture Notes in Computer Science, A. GasparCunha, C. Henggeler Antunes, and C. C. Coello, Eds. Springer International Publishing, 2015, vol. 9018, pp. 277-291.

[35] C. Coello and G. Lamont, Applications of Multi-objective Evolutionary Algorithms, ser. Advances in natural computation. World Scientific, 2004.

[36] C. A. C. Coello, "A Comprehensive Survey of Evolutionary-Based Multiobjective Optimization Techniques," Knowledge and Information Systems, vol. 1, pp. 269-308, 1999.

[37] C. A. Coello Coello, G. B.Lamont, and D. A. Veldhuizen, Evolutionary Algorithms for Solving Multi-Objective Problems. Kluwer Academic Publishers, New York,, March 2002.

[38] Y. Collette and P. Siarry, Multiobjective Optimization: Principles and Case Studies. Springer Science \& Business Media, 2003.

[39] K. Deb, L. Thiele, M. Laumanns, and E. Zitzler, "Scalable MultiObjective Optimization Test Problems," In Congress on Evolutionary Computation (CEC2002), Piscataway, New Jersey: IEEE service Center, vol. 1, pp. 825-830, MAy 2002.

[40] _ - "Scalable Test Problems for Evolutionary Multi-Objective Optimization," in Evolutionary Multiobjective Optimization: Theoretical Advances and Applications, A. Abraham, R. Jain, and R. Goldberg, Eds. Springer, 2005, ch. 6, pp. 105-145.

[41] K. Deb, Multi-Objective Optimization Using Evolutionary Algorithms, 2nd ed., S. Ross and R. Weber, Eds. John Wiley and Sons Ltd, 2002.

[42] K. Deb, A. Pratap, S. Agarwal, and T.Meyarivan, "A Fast and Elitist Multiobjective Genetic Algorithm:NSGA-II," IEEE Transsation On Evolutionary Computation, vol. 6, no. 2, pp. 182-197, 2002.

[43] D. Debels and M. Vanhoucke, "A Decomposition-Based Genetic Algorithm for the Resource-Constrained Project-Scheduling Problem," Operations Research, vol. 55, no. 3, pp. 457-469, 2007.

[44] B. Derbel, A. Liefooghe, G. Marquet, and E.-G. Talbi, "A Fine Grained Message Passing MOEA/D," in IEEE Congress on Evolutionary Computation (CEC'15), 2015, pp. 1837-1844.

[45] J. D.Knowles, "Local-Search and Hybrid Evolutionary Algorithms for Pareto Optimization," PhD Thesis, Department of Computer Science, University of Reading, Reading, RG6 6AY, UK., 2002.

[46] J. Dubois-Lacoste, M. Lpez-Ibez, and T. Sttzle, "Adaptive Anytime Two-Phase Local Search," vol. 6073, pp. 52-67.

[47] —- "Improving the Anytime Behavior of Two-Phase Local Search," Annals of Mathematics and Artificial Intelligence, vol. 61, no. 2, pp. 125-154, 2011.

[48] J. Durillo, Q. Zhang, A. Nebro, and E. Alba, "Distribution of Computational Effort in Parallel MOEA/D," in Learning and Intelligent Optimization, ser. Lecture Notes in Computer Science, C. Coello, Ed. Springer Berlin Heidelberg, 2011, vol. 6683, pp. 488-502.

[49] R. Eberhart and J.Kennedy, "A New Optimizer Using Particle Swarm Theory," in Proceedings of the Sixth International Symposium on Micro Machine and Human Science, MHS'95, oct. 1995, pp. 39-43.

[50] M. Fleischer, "The Measure of Pareto Optima Applications to Multiobjective Metaheuristics," in Evolutionary Multi-Criterion Optimization, ser. Lecture Notes in Computer Science, C. Fonseca, P. Fleming, E. Zitzler, L. Thiele, and K. Deb, Eds. Springer Berlin Heidelberg, 2003, vol. 2632, pp. 519-533.

[51] J. Fliege, L. M. G. Drummond, and B. F. Svaiter, "Newton's method for multiobjective optimization," SIAM Journal on Optimization, vol. 20, no. 2, pp. 602-626, 2009.

[52] C. Fonseca and P. Fleming, "An Overview of Evolutionary Algorithm in Multi-Objective Optimization," Evolutionary Computation, vol. 3, no. 1, pp. 1-16, 1995. 
[53] P. M. França, A. Mendes, and P. Moscato, "A Memetic Algorithm for the total Tardiness Single Machine Scheduling Problem," European Journal of Operational Research, vol. 132, no. 1, pp. 224-242, 2001.

[54] X. Gandibleux, N. Mezdaoui, and A. Frville, "A Tabu Search Procedure to Solve MultiObjective Combinatorial Optimization Problems," in Advances in Multiple Objective and Goal Programming, ser. Lecture Notes in Economics and Mathematical Systems, R. Caballero, F. Ruiz, and R. Steuer, Eds. Springer Berlin Heidelberg, 1997, vol. 455, pp. 291-300.

[55] M. Gong, F. Liu, W. Zhang, L. Jiao, and Q. Zhang, "Interactive MOEA/D for Multi-objective Decision Making," in Proceedings of the 13th annual conference on Genetic and evolutionary computation. ACM, 2011, pp. 721-728.

[56] C. Grosan, "Multiobjective Adaptive Representation Evolutionary Algorithm (MAREA)- A new Evolutionary Algorithm for Multiobjective Optimization." in WSC, ser. Advances in Soft Computing, A. Abraham, B. D. Baets, M. Kppen, and B. Nickolay, Eds., vol. 34. Springer, 2004, pp. 113-121.

[57] Guerra-Gomez, E. Tlelo-Cuautle, T. McConaghy, LuisG.delaFraga, G. Gielen, G.Reyes-Salgado, and J.M.Munoz-Pacheco, "Sizing Mixedmode Circuits by Multi-objective Evolutionary Algorithms," in 53rd IEEE International Midwest Symposium on Circuits and Systems, 2010, pp. 813-816.

[58] C. Guo, J. Zhibin, H. Zhang, and N. Li, "Decomposition-based classified Ant Colony Optimization Algorithm for Scheduling Semiconductor Wafer Fabrication System," Computers \& Industrial Engineering, vol. 62 , no. 1 , pp. 141-151, 2012

[59] M. P. Hansen and A. Jaszkiewicz, "Evaluating the Quality of Approximations to the Non-Dominated Set," Technical University of Denmark, Technical Report MM-REP-1998-7, 1998.

[60] S. Harris and E. Ifeachor, "Automatic Fesign of Frequency Sampling Filters by Hybrid Genetic Algorithm Techniques," IEEE Transactions on Signal Processing, vol. 46, no. 12, pp. 3304-3314, 1998.

[61] W. E. Hart, N. Krasnogor, and J. E. Smith, Recent advances in Memetic Algorithms. Springer Science \& Business Media, 2005, vol. 166.

[62] R. Hedayatzadeh, B. Hasanizadeh, R. Akbari, and K. Ziarati, "A Multi-Objective Artificial Bee Colony for Optimizing Multi-Objective Problems," in Advanced Computer Theory and Engineering (ICACTE), 2010 3rd International Conference on, vol. 5. IEEE, 2010, pp. V5277.

[63] T. Y. Hisao Ishibuchi and T. Murata, "Balance between Genetic Search and Local Search in Memetic Algorithms for Multiobjective Permutation Flowshop Scheduling," IEEE Transiction on Evolutionary Computation, vol. 7, no. 2, pp. 204-223, April 2003.

[64] R. Hooke and T. A. Jeeves, "Direct Search Solution of Numerical and Statistical Problems," Journal of ACM, vol. 8, pp. 212-229, April 1961.

[65] J. Horn, N. Nafpliotis, and D. E. Goldberg., "A Niched Pareto Genetic Algorithm for Multiobjective Optimization," in Proceedings of the First IEEE Conference on Evolutionary Computation, CEC'94, 1994.

[66] H. Ishibuchi, N. Akedo, and Y. Nojima, "Behavior of Multiobjective Evolutionary Algorithms on Many-Objective Knapsack Problems," IEEE Transactions on Evolutionary Computation, vol. 19, no. 2, pp. 264-283, 2015

[67] H. Ishibuchi and T. Murata, "Multi-Objective Genetic Local Search Algorithm and Its Application to Flowshop Scheduling," IEEE Transactions on Systems, Man and Cybernetics, vol. 28, no. 3, pp. 392-403, 1998.

[68] —, "Multi-objective Genetic Local Search for Minimizing the number of Fuzzy Rules for Pattern Classification Problems," in The 1998 IEEE International Conference on Fuzzy Systems Proceedings, vol. 2, 1998, pp. 1100-1105.

[69] H. Ishibuchi, N. Tsukamoto, and Y. Nojima, "Iterative Approach to Indicator Based Multiobjective Optimization," in Evolutionary Computation (CEC'07), 2007, pp. 3967-3974.

[70] H. Ishibuchi and T. Murata., "Multi-Objective Genetic Local Search Algorithm." in Proceedings of the Third IEEE International Conference on Evolutionary Computation, I. T. Fukuda and T. Furuhashi, Eds., Nagoya, Japan, 1996, pp. 119-124.

[71] H. Ishibuchi, Y. Sakane, N. Tsukamoto, and Y. Nojima, "Adaptation of
Scalarizing Functions in MOEA/D: An Adaptive Scalarizing FunctionBased Multiobjective Evolutionary Algorithm," in Proceedings of Evolutionary Multi-Criterion Optimization, 5th International Conference EMO'09, Nantes, France, April 7-10, 2009., 2009, pp. 438-452.

[72] —, "Effects of using two Neighborhood Structures on the Performance of Cellular Evolutionary Algorithms for Many-objective Optimization," in Proceedings of the IEEE Congress on Evolutionary Computation, CEC'09,Trondheim, Norway, 18-21 May, 2009, 2009, pp. $2508-2515$.

[73] _ "Evolutionary Many-Objective Optimization by NSGA-II and MOEA/D with Large Populations," in Proceedings of the IEEE International Conference on Systems, Man and Cybernetics, San Antonio, TX, USA, 11-14 October 2009, 2009, pp. 1758-1763.

[74] _ "Simultaneous use of Different Scalarizing Functions in MOEA/D," in Genetic and Evolutionary Computation Conference, GECCO'10, Proceedings, Portland, Oregon, USA, July 7-11, 2010, 2010, pp. 519-526.

[75] H. Ishibuchi, N. Tsukamoto, and Y. Nojima, "Evolutionary Manyobjective Optimization: A Short Review," in Proceedings of the IEEE Congress on Evolutionary Computation(CEC'08), Hong Kong, China, 2008, pp. 2419-2426.

[76] H. Ishibuchi, N. Tsukamoto, Y. Sakane, and Y. Nojima, "Indicatorbased Evolutionary Algorithm with Hypervolume Approximation by Achievement Scalarizing Functions," in 2010, Proceedings of Genetic and Evolutionary Computation Conference GECCO Portland, Oregon, USA, July 7-11, 2010, 2010, pp. 527-534.

[77] A. Jaszkiewicz, "On the Computational Efficiency of Multiple Objective Metaheuristics. The Knapsack Problem Case Study," European Journal of Operational Research, vol. 158, no. 2, pp. 418-433, 2004.

[78] A. Jaszkiewicz, M. Hapke, and P. Kominek, "Performance of Multiple Objective Evolutionary Algorithms on a Distribution System Design Problem-Computational Experiment," in Proceedings of First International Conference on Evolutionary Multi-Criterion Optimization (EMO), Zurich, Switzerland, March 7-9, 2001, pp. 241-255.

[79] S. Jiang, J. Zhang, Y.-S. Ong, A. Zhang, and P. S. Tan, "A Simple and Fast Hypervolume Indicator-Based Multiobjective Evolutionary Algorithm," IEEE Transactions on Cybernetics, vol. 45, no. 10, pp. 2202-2213, 2015.

[80] K.Deb, "Multiobjective Genetic Algorithms: Problems Difficulities and Construction of Test Problems," Evolutionary Computation, vol. 7, no. 3, pp. 205-230, 1999.

[81] K.Deb and R. Agrawal, "Simulated Binary Crossover for Continuous Search Space," Complex System, vol. 9, pp. 115-148, 1995.

[82] L. Ke, Q. Zhang, and R. Battiti, "MOEA/D-ACO: A Multiobjective Evolutionary Algorithm using Decomposition and AntColony Optimization," IEEE T. Cybernetics, vol. 43, no. 6, pp. 1845-1859, 2013.

[83] L. Ke, Q. Zhang, and R.Battiti, "Hybridization of Decomposition and Local Search for Multiobjective Optimization," IEEE Transactions on Cybernetics, vol. 44, no. 10, pp. 1808-1820, 2014.

[84] W. Khan, "Hybrid multiobjective evolutionary algorithm based on decomposition," PhD, Department of Mathematical Sciences, University of Essex, Wivenhoe Park, CO4 3SQ, Colchester, UK, January 2012.

[85] W. Khan, A. Salhi, M. A. Jan, and R. Khanum, "Enhanced Version of Gentically Adaptive Multi-Algorithm for Multiobjective Optimization," International Journal of Advanced Computer Science and Application (IJACSA), vol. 12, no. 6, pp. 279-287, 2015.

[86] W. Khan and Q. Zhang, “ MOEA/D-DRA with Two Crossover Operators," in Proceedings of the UK Workshop on Computational Intelligence (UKCI 2010), 8th-10th September 2010, pp. 1-6.

[87] V. Khare, X. Yao, and K. Deb, "Performance scaling of multiobjective evolutionary algorithms," in Evolutionary Multi-Criterion Optimization, ser. Lecture Notes in Computer Science, C. Fonseca, P. Fleming, E. Zitzler, L. Thiele, and K. Deb, Eds. Springer Berlin Heidelberg, 2003, vol. 2632, pp. 376-390.

[88] J. Knowles and D. Corne, "The Pareto Archived Evolution Strategy: A new Baseline Algorithm for Pareto Multiobjective Optimization," in Proceedings of the IEEE Congress on Evolutionary Computation (CEC' 99), Piscatay, NJ, JULY 1999, pp. 98-105.

[89] P. Koch, O. Kramer, G. Rudolph, and N. Beume, "On the hybridization of sms-emoa and local search for continuous multiobjective optimiza- 
tion," in Proceedings of the 11th Annual Conference on Genetic and Evolutionary Computation, ser. GECCO '09. New York, NY, USA: ACM, 2009, pp. 603-610.

[90] J. Kocijan, R. Murray-Smith, C. Rasmussen, and A. Girard, "Gaussian Process Model based Predictive Control," in Proceedings of theAmerican Control Conference, vol. 3, 2004, pp. 2214-2219.

[91] A. Konstantinidis, C. Charalambous, A. Zhou, and Q. Zhang, "Multi-objective Mobile Agent-based Sensor Network Routing using MOEA/D," in Evolutionary Computation (CEC), 2010 IEEE Congress on. IEEE, 2010, pp. 1-8.

[92] A. Konstantinidis, K. Yang, and Q. Zhang, "Problem-specific Encoding and Genetic Operation for a Multi-Objective Deployment and Power Assignment Problem in Wireless Sensor Networks," in IEEE International Conference on Communications. IEEE, 2009, pp. 1-6.

[93] A. Konstantinidis, K. Yang, Q. Zhang, and F. Gordejuela-Sanchez, "Multiobjective K-connected Deployment and Power Assignment in WSNs using Constraint Handling," in IEEE Global Telecommunications Conference (GLOBECOM'09). IEEE, 2009, pp. 1-6.

[94] N. Krasnogor, A. Aragn, and J. Pacheco, "Memetic Algorithms," in Metaheuristic Procedures for Training Neutral Networks, ser. Operations Research/Computer Science Interfaces Series, E. Alba and R. Mart, Eds. Springer US, 2006, vol. 36, pp. 225-248.

[95] M. Laumanns, E. Zitzler, and L. Thiele, "A Unified Model For MultiObjective Evolutionary Algorithms with Elitism," in Proceedings of the 2000 Congress on Evolutionary Computation, vol. 1, 2000, pp. $46-53$ vol.1.

[96] C. Lcken, B. Barn, and C. Brizuela, "A Survey on Multi-Objective Evolutionary Algorithms for Many-objective Problems," Computational Optimization and Applications, vol. 58, no. 3, pp. 707-756, 2014.

[97] H. Li and D. Landa-Silva, "An Adaptive Evolutionary Multi-objective Approach Based on Simulated Annealing," Evolutionary Computing, vol. 19 , no. 4, pp. 561-595, Dec. 2011.

[98] H. Li, "Combination of Evolutionary Algorithms with Decomposition Techniques for Multiobjective Optimization," PhD, Department of Computer Science, University of Essex, Wevehoe Park, Colchester, Essex, CO4 3SQ, UK, 2007.

[99] H. Li, M. Ding, J. Deng, and Q. Zhang, "On the use of Random Weights in MOEA/D," in IEEE Congress on Evolutionary Computation (CEC'15), 2015, pp. 978-985.

[100] H. Li, X. Su, Z. Xu, and Q. Zhang, "MOEA/D with Iterative Thresholding Algorithm for Sparse Optimization Problems," in Parallel Problem Solving from Nature - PPSN XII, ser. Lecture Notes in Computer Science, C. Coello, V. Cutello, K. Deb, S. Forrest, G. Nicosia, and M. Pavone, Eds. Springer Berlin Heidelberg, 2012, vol. 7492, pp. 93-101.

[101] H. Li and Q. Zhang, "Multiobjective Optimization Problems With Complicated Pareto Sets: MOEA/D and NSGA-II," IEEE Transsation On Evolutionary Computation, vol. 13, no. 2, pp. 284-302, April 2009.

[102] _ _ "A Decomposition-based Evolutionary Strategy for Bi-objective LOTZ Problem," in Adaptation in Artificial and Biological Systems. University of Essex, UK., April 2006, pp. 1-5.

[103] _ _ "A Multiobjective Differential Evolution Based on Decomposition for Multiobjective Optimization with Variable Linkages," in Parallel Problem Solving from Nature - PPSN IX, ser. Lecture Notes in Computer Science, T. Runarsson, H.-G. Beyer, E. Burke, J. MereloGuervs, L. Whitley, and X. Yao, Eds. Springer Berlin Heidelberg, 2006, vol. 4193, pp. 583-592.

[104] K. Li, K. Deb, and Q. Zhang, "Evolutionary Multiobjective Optimization with Hybrid Selection Principles," in IEEE Congress on Evolutionary Computation (CEC'15), 2015, pp. 900-907.

[105] K. Li, K. Deb, Q. Zhang, and S. Kwong, "An Evolutionary ManyObjective Optimization Algorithm Based on Dominance and Decomposition," IEEE Transactions on Evolutionary Computation, vol. 19, no. 5, pp. 694-716, October 2015.

[106] K. Li, Á. Fialho, S. Kwong, and Q. Zhang, "Adaptive Operator Selection With Bandits for a Multiobjective Evolutionary Algorithm Based on Decomposition," IEEE Trans. Evolutionary Computation, vol. 18, no. 1, pp. 114-130, 2014
[107] K. Li, S. Kwong, Q. Zhang, and K. Deb, "Interrelationship-Based Selection for Decomposition Multiobjective Optimization," IEEE Transactions on Cybernetics, vol. 45, no. 10, pp. 2076-2088, 2015.

[108] K. Li, Q. Zhang, S. Kwong, M. Li, and R. Wang, "Stable MatchingBased Selection in Evolutionary Multiobjective Optimization," IEEE Transactions on Evolutionary Computation, vol. 18, no. 6, pp. 909923, 2014.

[109] X. Liao, Q. Li, X. Yang, W. Zhang, and W. Li, "Multiobjective Optimization for Crash Safety Design of Vehicles using Stepwise Regression Model," Structural and Multidisciplinary Optimization, vol. 35, no. 6, pp. 561-569, 2008.

[110] H. lin Liu, F. Gu, and Y. ming Cheung, "T-MOEA/D: MOEA/D with Objective Transform in Multi-objective Problems," in International Conference of Information Science and Management Engineering (ISME'10), vol. 2, 2010, pp. 282-285.

[111] Y. Liu and B. Niu, "A Multi-objective Particle Swarm Optimization Based on Decomposition," in Emerging Intelligent Computing Technology and Applications, ser. Communications in Computer and Information Science, D.-S. Huang, P. Gupta, L. Wang, and M. Gromiha, Eds. Springer Berlin Heidelberg, 2013, vol. 375, pp. 200-205.

[112] F. Lootsma and K. Ragsdell, "State-of-the-art in Parallel Nonlinear Optimization," Parallel Computing, vol. 6, no. 2, pp. 133-155, 1988.

[113] T. Lust and A. Jaszkiewicz, "Speed-up Techniques for solving largescale biobjective TSP," Computers \& Operations Research, vol. 37 , no. 3, pp. 521-533, 2010.

[114] P. H. Lyndon While and S. Huband, "A Faster Algorithm for Calculating Hypervolume," IEEE Transactions on Evolutionary Computation, vol. 10, no. 1, pp. 29-38, February 2006.

[115] X. Ma, F. Liu, Y. Qi, M. Gong, M. Yin, L. J. Lingling Li, and J. Wu, "MOEA/D with Opposition-Based Learning for Multiobjective Optimization Problem," Neurocomputing, vol. 146, pp. 48-64, 2014.

[116] X. Ma, Y. Qi, L. Li, F. Liu, L. Jiao, and J. Wu, "MOEA/D with Uniform Decomposition Measurement for Many-Objective Problems," Soft Computing, vol. 18, no. 12, pp. 2541-2564, 2014.

[117] A. Mambrini and D. Izzo, "PaDe: A Parallel Algorithm Based on the MOEA/D Framework and the Island Model," in Proceedings OF 13th International Conference Parallel Problem Solving from Nature-PPSN XIII, Ljubljana, Slovenia, September 13-17, 2014., 2014, pp. 711-720.

[118] I. B. Mansour and I. Alaya, "Indicator Based Ant Colony Optimization for Multi-objective Knapsack Problem," Procedia Computer Science, vol. 60, pp. 448-457, 2015.

[119] G. Marquet, B. Derbel, A. Liefooghe, and E. Talbi, "Shake Them All! - Rethinking Selection and Replacement in MOEA/D," in Proceedings of Parallel Problem Solving from Nature-PPSN XIII-13th International Conference, Ljubljana, Slovenia, September 13-17, 2014, pp. 641-651.

[120] S. Z. Martnez and C. A. C. Coello, "A Hybridization of MOEA/D with the Nonlinear Simplex Search Algorithm," in IEEE Congress on Evolutionary Computation (CEC'13), 2013, pp. 48-55.

[121] W. K. Mashwan, "Enhanced versions of Differential Evolution: Stateof-the-art Survey," International Journal Computing Sciences and Mathematics(IJCSM), vol. 5, no. 2, pp. 107-126, 2014.

[122] W. K. Mashwani, "A Multimethod Search Approach Based on Adaptive Generations Level," in Seventh International Conference on Natural Computation(ICNC'11), Shanghai, China, 26-28 July, 2011, pp. 23-27.

[123] _ - "Hybrid Multiobjective Evolutionary Algorithms: A Survey of the State-of-the-art," International Journal of Computer Science Issues, vol. 8, no. 6, pp. 374-392, 2011.

[124] _ _ "Integration of NSGA-II and MOEA/D in Multimethod Search Approach: Algorithms," in Proceedings of the 13th Annual Conference Companion on Genetic and Evolutionary Computation. ACM, 2011, pp. $75-76$.

[125] — "MOEA/D with DE and PSO: MOEA/D-DE+PSO," in The Thirty-first SGAI International Conference on Innovative Techniques and Applications of Artificial Intelligence, Cambridge, UK, December, 2011, pp. 217-221.

[126] _ "Comparison of Evolutionary Algorithm over Multiobjective Optimization Problems," in Proceeding of International Conference on Modeling and Simulation (ICOMS), Air University Islamabad, Pakistan, 2013. 
[127] — "Comprehensive Survey of the Hybrid Evolutionary Algorithms," International Journal of Applied Evolutionary Computation (IJAEC), vol. 4, no. 2, pp. 1-19, July 2013.

[128] _ "Performanance of AMALGAM over CEC'09 Test Instances,' in Proceeding Third International Conference on Aerospace Science and Engineering (ICASE'13), 2013.

[129] W. K. Mashwani and A. Salhi, "A Decomposition-Based Hybrid Multiobjective Evolutionary Algorithm with Dynamic Resource Allocation," Applied Soft Computing, vol. 12, no. 9, pp. 2765-2780, 2012.

[130] _ _ "Multiobjective Evolutionary Algorithm Based on Multimethod with Dynamic Resources Allocation," Applied Soft Computing, vol. 39, pp. 292-309, 2016.

[131] W. K. Mashwani, A. Salhi, M. A. Jan, R.A.Khanum, and M. Sulaiman, "Impact Analysis of Crossovers in Multiobjective Evolutionary Algorithm," Science International Journal, vol. 27, no. 6, pp. 4943-4956, December 2015.

[132] W. K. Mashwani and A. Salhi, "Multiobjective Memetic Algorithm Based on Decomposition," Applied Soft Computing, vol. 21, pp. 221243, 2014.

[133] M. Medina, S. Das, C. Coello Coello, and J. Ramirez, "Two decomposition-based modem metaheuristic algorithms for multiobjective optimization- A comparative study," in Computational Intelligence in Multi-Criteria Decision-Making (MCDM), 2013 IEEE Symposium on, 2013, pp. 9-16.

[134] Y. Mei, K. Tang, and X. Yao, "Decomposition-Based Memetic Algorithm for Multiobjective Capacitated Arc Routing Problem," IEEE Trans. Evolutionary Computation, vol. 15, no. 2, pp. 151-165, 2011.

[135] _ _ "Decomposition-based memetic algorithm for multiobjective capacitated arc routing problem," Evolutionary Computation, IEEE Transactions on, vol. 15, no. 2, pp. 151-165, 2011.

[136] K. M. Miettinien, Nonlinear Multiobjective Optimization, ser Kluwer's International Series. Norwell, MA: Academic Publishers Kluwer, 1999.

[137] P. Moscato, A. Mendes, and C. Cotta, Memetic Algorithms. New Optimization Techniques in Engineering. Berlin Heidelberg: Springer, 2004.

[138] P. Moscato, "On Evolution, Search, Optimization, Genetic Algorithms and Martial Arts-Towards Memetic Algorithms," Caltech Concurrent Computation Program, C3P Report, vol. 826, 1989.

[139] _ - "Memetic Algorithms: A Short Introduction," in New Ideas in Optimization. McGraw-Hill Ltd., UK, 1999, pp. 219-234.

[140] P. Moscato and M. G. Norman, "A Memetic Approach for the Traveling Salesman Problem Implementation of a Computational Ecology for Combinatorial Optimization On Message-passing Systems," Parallel Computing and Transputer Applications, vol. 1, pp. 177-186, 1992.

[141] T. Murata and M. Gen, "Cellular Genetic Algorithm for MultiObjective Optimization," in In Proc. of the 4th Asian Fuzzy System Symposium, 2002, pp. 538-542.

[142] T. Murata, H. Ishibuchi, and M. Gen, "Specification of Genetic Search Direction in Cellular Multiobjective Genetic Algorithm," in Evolutionary Multicriterion Optimization. LNCS, Springer-Verlag, pp. 82-95.

[143] M. Nasir, A. K. Mondal, S. Sengupta, S. Das, and A. Abraham, "An Improved Multiobjective Evolutionary Algorithm based on Decomposition with Fuzzy Dominance," in Proceedings of IEEE Congress on Evolutionary Computation $(C E C, 11)$. New Orleans, US: IEEE Press, June 5-8 2011, pp. 1-8.

[144] A. J. Nebro, J. J. Durillo, F. Luna, B. Dorronsoro, and E. Alba, "MOCell: A Cellular Genetic Algorithm for Multiobjective Optimization," International Journal of Intelligent Systems, pp. 25-36, 2007.

[145] A. Nebro and J. Durillo, "A Study of the Parallelization of the Multi-Objective Metaheuristic MOEA/D," in Learning and Intelligent Optimization, ser. Lecture Notes in Computer Science, C. Blum and R. Battiti, Eds. Springer Berlin Heidelberg, 2010, vol. 6073, pp. 303-317.

[146] F. Neri and C. Cotta, "Memetic Algorithms and Memetic Computing Optimization: A literature Review," Swarm and Evolutionary Computation, vol. 2, pp. 1-14, 2012.

[147] F. Neri, C. Cotta, and P. Moscato, Handbook of Memetic Algorithms. Springer Heidelberg, 2012, vol. 379.
[148] T. Niknam, F. Golestaneh, and M. Sadeghi, "Multiobjective Teaching Learning-Based Optimization for Dynamic Economic Emission Dispatch," Systems Journal, IEEE, vol. 6, no. 2, pp. 341-352, 2012.

[149] N.Srinivas and K.Deb, "A Multiobjective Optimization using Nondominated Sorting in Genetic Algorithms," J Evol Comput, vol. 2, no. 3 , pp. 221-248, 1994.

[150] Y. S. Ong and A. Keane, "Meta-Lamarckian learning in Lemetic Algorithms," IEEE Transactions on Evolutionary Computation, vol. 8 , no. 2, pp. 99-110, 2004.

[151] Y.-S. Ong, M. H. Lim, and X. Chen, "Research frontier-memetic computationpast, present \& future," IEEE Computational Intelligence Magazine, vol. 5, no. 2, p. 24, 2010.

[152] S. Pal, B.-Y.Qu, S. Das, and P.N.Suganthan, "Optimal Synthesis of Linear Antenna Arrays with Multi-Objective Differential Evolution," Progress In Electromagnetics Research B, vol. 21, pp. 87-111, 2010.

[153] S. Pal, S. Das, A. Basak, and P. Suganthan, "Synthesis of Difference Patterns for Monopulse Antennas with Optimal Combination of array-size and number of subarrays-A Multi-Objective Optimization Approach," Progress In Electromagnetics Research B, vol. 21, pp. 257-280, 2010.

[154] P. Palmers, T. McConnaghy, M. Steyaert, and G. Gielen, "Massively Multi-topology Sizing of analog Integrated Circuits," in Proceedings of the Conference on Design, Automation and Test in Europe (DATE'09). 3001 Leuven, Belgium: European Design and Automation Association, 2009, pp. 706-711.

[155] L. Paquete and T. Stützle, "A Two-Phase Local Search for the Biobjective Traveling Salesman Problem," in Proceedings of the Evolutionary Multi-Criterion Optimization, Second International Conference, EMO'3, Faro, Portugal, April 8-11, 2003, pp. 479-493.

[156] W. Peng and Q. Zhang, "A Decomposition-based Multi-objective Particle Swarm Optimization Algorithm for Continuous Optimization Problems," in IEEE International Conference on Granular Computing, 2008, pp. 534-537.

[157] D. Phan and J. Suzuki, "R2-IBEA: R2 indicator based evolutionary algorithm for multiobjective optimization," in IEEE Congress on Evolutionary Computation (CEC'13), 2013, pp. 1836-1845.

[158] M. Pilát and R. Neruda, "Hypervolume-based Local Search in Multiobjective Evolutionary Optimization," in Proceedings of the Annual Conference on Genetic and Evolutionary Computation, ser. GECCO '14. New York, NY, USA: ACM, 2014, pp. 637-644.

[159] R. C. Purshouse and P. J. Fleming, "Evolutionary Many-Objective Optimisation: An Exploratory Analysis," in IEEE Congress on Evolutionary Computation (CEC'03), vol. 3. IEEE, 2003, pp. 2066-2073.

[160] Y. Qi, Z. Hou, H. Li, J. Huang, and X. Li, "A decomposition based memetic algorithm for multi-objective vehicle routing problem with time windows," Computers and Operations Research, vol. 62, no. C, pp. 61-77, October 2015.

[161] Y. Qi, X. Ma, L. J. Fang Liu, J. Sun, and J. Wu, "MOEA/D with Adaptive Weight Adjustment," Evolutionary Computation, vol. 22, no. 2, pp. 231-264, May 2014.

[162] R.Storn and K.V.Price, "Differential Evolution - A Simple and Efficient Heuristic for Global Optimization over Continuous Spaces," ICSI, Technical Report TR-95-012, 1995.

[163] 1. Rubio-Largo, Q. Zhang, and M. Vega-Rodrguez, "Multiobjective Evolutionary Algorithm Based On Decomposition for 3-objective Optimization Problems with Objectives in Different Scales," Soft Computing, vol. 19, no. 1, pp. 157-166, 2015.

[164] H. Sato, M. Miyakawa, and E. Pérez-Cortés, "A Parallel MOEA/D Generating Solutions in Minimum Overlapped Update Ranges of Solutions," in Proceedings of the Companion Publication of the 2015 Annual Conference on Genetic and Evolutionary Computation, ser. GECCO Companion '15. New York, NY, USA: ACM, 2015, pp. 775-776.

[165] A. Schuster and Würzburg, "About Travelling Salesmen and Telephone Network-Combinatirial Optimization at High School," ZDM international Reviwer on Mathematical Education, vol. 36, no. 2, pp. 77-81, 2004.

[166] R. Shang, J. Wang, L. Jiao, and Y. Wang, "An Improved Decomposition-Based Memetic Algorithm for Multi-Objective Capac- 
itated Arc Routing Problem,” Applied Soft Computing, vol. 19, pp. 343-361, 2014.

[167] D. Simon, Evolutionary Optimization Algorithms: Biologically Inspired and Population-Based Approches to Computer Intelligence. John Wiley \& Sons, 2013.

[168] V. A. Sosa-Hernandez, O. Schütze, G. Rudoph, and H. Trautmann, "Directed search method for indicator-based multi-objective evolutionary algorithms," in Proceedings of the 15th Annual Conference Companion on Genetic and Evolutionary Computation, ser. GECCO '13 Companion. New York, NY, USA: ACM, 2013, pp. 1699-1702.

[169] K. Srensen, "Metaheuristicsthe Metaphor Exposed," International Transactions in Operational Research, vol. 22, no. 1, pp. 3-18, 2015.

[170] M. G. C. Tapia and C. A. C. Coello, "Applications of Multi-Objective Evolutionary Algorithms in Economics and Finance: A Survey." IEEE Congress on Evolutionary Computation, vol. 7, pp. 532-539, 2007.

[171] L. Thiele, K. Miettinen, P. J. Korhonen, and J. Molina, "A PreferenceBased Evolutionary Algorithm for Multi-Objective Optimization," Evolutionary Computation, vol. 17, no. 3, pp. 411-436, 2009.

[172] H. Tizhoosh, "Opposition-Based Learning: A New Scheme for Machine Intelligence," in Computational Intelligence for Modelling, Control and Automation, 2005 and International Conference on Intelligent Agents, Web Technologies and Internet Commerce, International Conference on, vol. 1, 2005, pp. 695-701.

[173] H. Trautmann, T. Wagner, D. Biermann, and C. Weihs, "Indicatorbased Selection in Evolutionary Multiobjective Optimization Algorithms Based On the Desirability Index," Journal of Multi-Criteria Decision Analysis, vol. 20, no. 5-6, pp. 319-337, 2013.

[174] H. Trautmann, T. Wagner, and D. Brockhoff, "R2-EMOA: Focused Multiobjective Search Using R2-Indicator-Based Selection," in LION, ser. Lecture Notes in Computer Science, G. Nicosia and P. M. Pardalos, Eds., vol. 7997. Springer, 2013, pp. 70-74.

[175] D. Tuyttens, J. Teghem, P. Fortemps, and K. Nieuwenhuyze, "Performance of the MOSA Method for the Bicriteria Assignment Problem," Journal of Heuristics, vol. 6, no. 3, pp. 295-310, 2000.

[176] T. Ulrich, J. Bader, and L. Thiele, "Defining and optimizing indicatorbased diversity measures in multiobjective search," in Proceedings of the 11th international conference on Parallel problem solving from nature: Part I, ser. PPSN'10. Berlin, Heidelberg: Springer-Verlag, 2010, pp. 707-717.

[177] J. A. Vrugt and B. A. Robinson, "Improved Evolutionary Optimization from Genetically Adaptive Mutimethod Search," Proceedings of the National Academy of Sciences of the United States of America: PNAS (USA), vol. 104, no. 3, pp. 708-701, 16th Jaunuary 2007.

[178] M. Wagner, K. Bringmann, T. Friedrich, and F. Neumann, "Efficient Optimization of Many Objectives by Approximation-Guided Evolution," European Journal of Operational Research, vol. 243, no. 2, pp. 465-479, 2015

[179] T. Wagner, N. Beume, and B. Naujoks, "Pareto-, Aggregation-, and Indicator-Based Methods in Many-Objective Optimization," in Evolutionary Multi-Criterion Optimization, ser. Lecture Notes in Computer Science, S. Obayashi, K. Deb, C. Poloni, T. Hiroyasu, and T. Murata, Eds. Springer Berlin Heidelberg, 2007, vol. 4403, pp. 742-756.

[180] G. Wang, J. Chen, T. Cai, and B. Xin, "Decomposition-Based MultiObjective Differential Evolution Particle Swarm Optimization for The Design of a Tubular Permanent Magnet Linear Synchronous Motor," Engineering Optimization, vol. 45, no. 9, pp. 1107-1127, 2013.

[181] M. Woehrle, D. Brockhoff, T. Hohm, and S. Bleuler, "Investigating Coverage and Connectivity Trade-offs in Wireless Sensor Networks: The Benefits of MOEAs," in Multiple Criteria Decision Making for Sustainable Energy and Transportation Systems (MCDM 2008), ser. LNEMS, M. Ehrgott et al., Eds., vol. 634. Heidelberg, Germany: Springer, 2010, pp. 211-221.

[182] S. Zapotecas-Martínez, B. Derbel, A. Liefooghe, D. Brockhoff, H. E Aguirre, and K. Tanaka, "Injecting CMA-ES into MOEA/D," in Proceedings of the 2015 Annual Conference on Genetic and Evolutionary Computation, ser. GECCO '15. New York, NY, USA: ACM, 2015, pp. 783-790.

[183] Q. Zhang and H. Li, "MOEA/D: A Multiobjective Evolutionary Algorithm Based on Decomposition ," IEEE transaction on Evolutionary Computation, vol. 11, no. 6, pp. 712-731, December 2007.
[184] Q. Zhang, H. Li, D. Maringer, and E. P. K. Tsang, "MOEA/D with NBI-style Tchebycheff Approach for Portfolio Management," in Proceedings of the IEEE Congress on Evolutionary Computation, CEC 2010, Barcelona, Spain, 18-23 July 2010, 2010, pp. 1-8.

[185] Q. Zhang, W. Liu, and H. Li, "The Performance of a New Version of MOEA/D on CEC'09 Unconstrained MOP Test Instances," IEEE Congress On Evolutionary Computation (IEEE CEC 2009), Trondheim, Norway, pp. 203-208, May, 18-21 2009.

[186] Q. Zhang, W. Liu, E. Tsang, and B. Virginas, "Expensive multiobjective optimization by MOEA/D with Gaussian Process Model," Trans. Evol. Comp, vol. 14, pp. 456-474, June 2010.

[187] Q. Zhang, A. Zhou, S. Zhaoy, P. N. Suganthany, W. Liu, and S. Tiwariz, "Multiobjective Optimization Test Instances for the CEC 2009 Special Session and Competition," Technical Report CES-487, 2009.

[188] S.-Z. Zhao, P. N. Suganthan, and Q. Zhang, "Decomposition-Based Multiobjective Evolutionary Algorithm With an Ensemble of Neighborhood Sizes," IEEE Trans. Evolutionary Computation, vol. 16, no. 3, pp. 442-446, 2012.

[189] A. Zhou, B.-Y. Qu, H. Li, S.-Z. Zhao, P. N. Suganthan, and Q. Zhang., "Multiobjective evolutionary algorithms: A survey of the state-of-theart," Swarm and Evolutionary Computation, vol. 1, pp. 32-49, 16 March 2011, online publised.

[190] A. Zhou, Q. Zhang, and G. Zhang, "A Multiobjective Evolutionary Algorithm Based on Decomposition and Probability Model," in IEEE Congress on Evolutionary Computation (CEC'12). IEEE, 2012, pp. $1-8$.

[191] E. Zitzler, "Evolutionary Algorithms for Multiobjective Optimization Methods and Applications," Ph.D. dissertation, ETH Zurich, Switzerland, 1999.

[192] E. Zitzler, M. Laumanns, and L. Thiele, "SPEA2: Improving the Strength Pareto Evolutionary Algorithm," Computer Engineering and Networks Laboratory (TIK), ETH Zurich, Zurich, Switzerland, TIK Report 103, 2001.

[193] E. Zitzler and L. Thiele, "An Evolutionary Approach for Multiobjective Optimization: The Strength Pareto Approach," Computer Engineering and Networks Laboratory (TIK), ETH Zurich, TIK Report 43, May 1998.

[194] _ _ "An Evolutionary Approach for Multiobjective Optimization: The Strength Pareto Approach," Computer Engineering and Networks Laboratory (TIK), ETH Zurich, TIK Report 43, May 1998.

[195] _ - "Multiobjective Evolutionary Algorithms: A Comparative Case Study and the Strength Pareto Approach," IEEE Transactions on Evolutionary Computation, vol. 3, no. 4, pp. 257-271, November 1999.

[196] E. Zitzler, L. Thiele, and J. Bader, "SPAM: Set Preference Algorithm for Multiobjective Optimization," in Conference on Parallel Problem Solving From Nature (PPSN X), ser. LNCS, G. Rudolph et al., Eds., vol. 5199. Springer, 2008, pp. 847-858.

[197] E. Zitzler, L. Thiele, M. Laumanns, C. M. Fonseca, and V. Grunert da Fonseca, "Performance Assessment of Multiobjective Optimizers: An Analysis and Review," IEEE Transactions on Evolutionary Computation, vol. 7, no. 2, pp. 117-132, 2003.

[198] E. Zitzler, K. Deb, and L. Thiele, "Comparsion of Multiobjective Evolutionary Algorithms: Emperical Results," Evolutionary Computation, vol. 8, no. 2, pp. 173-195, 2000.

[199] E. Zitzler and S. Knzli, "Indicator-based Selection in Multiobjective Search," in in Proceeding of $8^{\text {th }}$ International Conference on Parallel Problem Solving from Nature (PPSN VIII. S Springer, 2004, pp. 832842.

[200] _ "Indicator-Based Selection in Multiobjective Search," in Parallel Problem Solving from Nature - PPSN VIII, ser. Lecture Notes in Computer Science, X. Yao, E. Burke, J. Lozano, J. Smith, J. MereloGuervs, J. Bullinaria, J. Rowe, P. Tino, A. Kabn, and H.-P. Schwefel, Eds. Springer Berlin Heidelberg, 2004, vol. 3242, pp. 832-842. 